$\mathrm{SPhT} / 99-126$

\title{
Meanders: Exact Asymptotics
}

\author{
P. Di Francesco, \\ O. Golinelli \\ and \\ E. Guitter*, \\ Service de Physique Théorique, C.E.A. Saclay, \\ F-91191 Gif sur Yvette, France
}

\begin{abstract}
We conjecture that meanders are governed by the gravitational version of a $c=-4$ two-dimensional conformal field theory, allowing for exact predictions for the meander configuration exponent $\alpha=\sqrt{29}(\sqrt{29}+\sqrt{5}) / 12$, and the semi-meander exponent $\bar{\alpha}=$ $1+\sqrt{11}(\sqrt{29}+\sqrt{5}) / 24$. This result follows from an interpretation of meanders as pairs of fully packed loops on a random surface, described by two $c=-2$ free fields. The above values agree with recent numerical estimates. We generalize these results to a score of meandric numbers with various geometries and arbitrary loop fugacities.
\end{abstract}

$10 / 99$

* e-mails: philippe,golinelli,guitter@spht.saclay.cea.fr 


\section{Introduction}

Meanders are a simply stated combinatorial problem consisting in counting the number $M_{n}$ of configurations of a closed self-avoiding road crossing an infinite river through a given number $2 n$ of bridges. Meanders appear in several domains of science including computer science [1], mathematics in connection with both Hilbert's 16th problem and the enumeration of ovals of planar algebraic curves [2] and the classification of 3-manifolds [3]. Meanders also appear in physics as a particular example of critical phenomena: indeed, meanders also count a particular class of Self-Avoiding Walks describing the compact foldings of a linear chain.

Among the various techniques used to attack the problem we can mention direct enumerations [4] [5] [6] [7], whose most recent one [8] enumerates up to $2 n=48$ bridges with a new transfer matrix method. Other approaches use random matrices [9] [10] [11] [12], or algebraic techniques based on the Temperley-Lieb algebra [13. Several exact results have been obtained for meander-related issues, such as exact sum rules for meandric numbers [10], and the calculation of a meander-related determinant [13] [3], but despite many efforts, no explicit formula for $M_{n}$ has been found so far for arbitrary $n$.

As a critical phenonemon, meanders are characterized by critical exponents describing the asymptotic behavior of $M_{n}$ for large $n$. We expect a behavior:

$$
M_{n} \underset{n \rightarrow \infty}{\sim} \frac{R^{2 n}}{n^{\alpha}}
$$

where $\log R$ is the entropy per bridge and $\alpha$ the configuration exponent. The best estimates extracted from extrapolation of finite $n$ exact results read $R^{2}=12.262874(15)$ and $\alpha=3.4206(4)[8]$.

In this paper, we present explicit formulas for the asymptotics of meanders based on a conjecture stating that meanders are governed by a two-dimensional conformal field theory with central charge $c=-4$ coupled to gravity. In particular, we obtain

$$
\alpha=\sqrt{29} \frac{\sqrt{29}+\sqrt{5}}{12}=3.42013288 \ldots
$$

in agreement with [8] and

$$
\bar{\alpha}=1+\sqrt{11} \frac{\sqrt{29}+\sqrt{5}}{24}=2.05319873 \ldots
$$


where $\bar{\alpha}$ is the configuration exponent describing the asymptotics for the semi-meander numbers $\bar{M}_{n}$ counting configurations of a closed self-avoiding road crossing a semi-infinite river (i.e a river with a source around which the road may wind) through $n$ bridges. Again this value is in agreement with the best estimate $\bar{\alpha}=2.056(10)$ found in [7].

Our conjecture is based on an interpretation of the meander problem as a pair of two fully packed loop models on a random surface, whose counterpart on a flat surface is a two-dimensional Coulomb gas whose critical behaviour is described by two decoupled $c=-2$ free fields.

The paper is organized as follows: In Section 2, we recall the $O\left(n_{1}, n_{2}\right)$ matrix model describing the generating function for meanders and semi-meanders with possibly several connected components of road and river, with a fugacity $n_{1}$ per river and $n_{2}$ per road. Section 3 identifies the matrix model as a fully packed loop problem on a random surface and discusses its flat counterpart, thus obtaining the central charge for arbitrary fugacities. In Section 4 , we extract the critical exponents $\alpha$ and $\bar{\alpha}$ for meanders and semi-meanders $\left(n_{1}, n_{2} \rightarrow 0\right)$ thanks to the KPZ formula [14] relating flat to random geometry. Section 5 presents several extensions of the configuration exponents corresponding to more involved river geometries. We conclude with more prospective results for arbitrary fugacities.

\section{Meanders and the $\mathbf{O}\left(n_{1}, n_{2}\right)$ matrix model}

As shown in [10], the meander problem can be formulated as a Hermitian matrix model, hereafter referred to as the $O\left(n_{1}, n_{2}\right)$ model, with $n_{1}$ black matrices $B_{1}, . ., B_{n_{1}}$ and $n_{2}$ white ones $W_{1}, \ldots, W_{n_{2}}$, all of size $N \times N$, with partition function

$$
\begin{aligned}
Z_{n_{1}, n_{2}}(N ; x) & =\int \prod_{i=1}^{n_{1}} d B_{i} \prod_{j=1}^{n_{2}} d W_{j} e^{-N \operatorname{Tr} V\left(\left\{B_{i}\right\},\left\{W_{j}\right\}\right)} \\
V\left(\left\{B_{i}\right\},\left\{W_{j}\right\}\right) & =\frac{1}{2}\left(\sum_{i=1}^{n_{1}} B_{i}^{2}+\sum_{j=1}^{n_{2}} W_{j}^{2}-x \sum_{i=1}^{n_{1}} \sum_{j=1}^{n_{2}} B_{i} W_{j} B_{i} W_{j}\right) .
\end{aligned}
$$

Expanding the planar free energy, we get

$$
\begin{aligned}
F_{n_{1}, n_{2}}(x) & =\lim _{N \rightarrow \infty} \frac{1}{N^{2}} \log Z_{n_{1}, n_{2}}(N ; x) \\
& =\sum_{n=1}^{\infty} x^{n} n_{1} n_{2} f_{n}\left(n_{1}, n_{2}\right)
\end{aligned}
$$



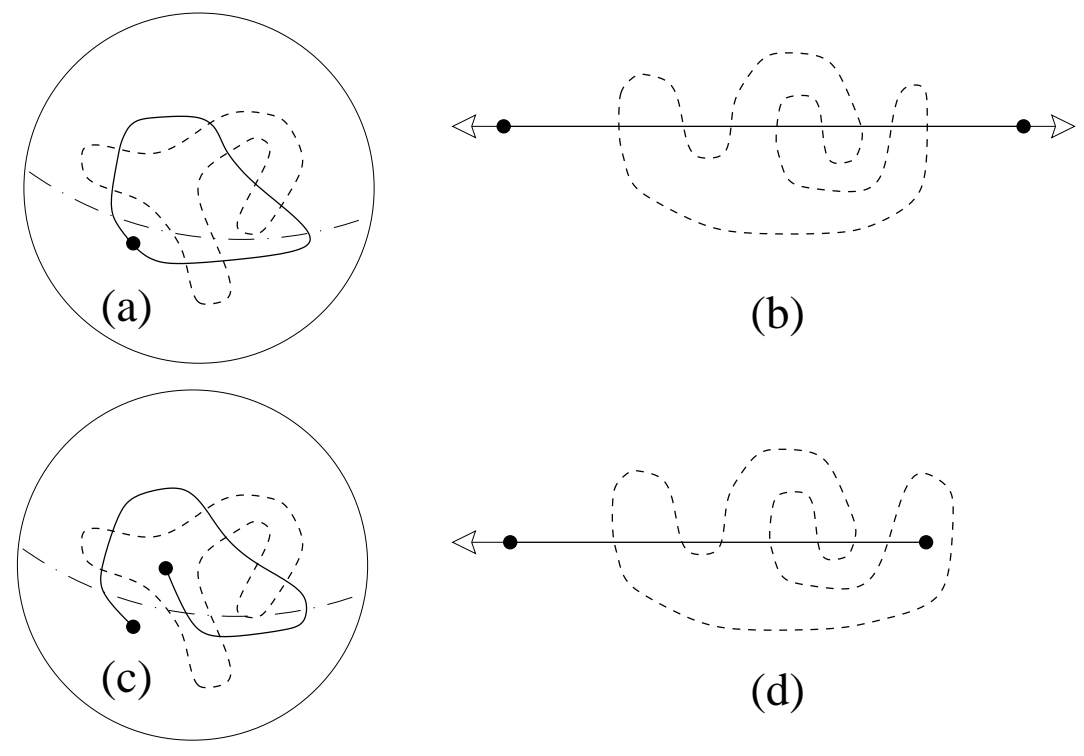

(b)

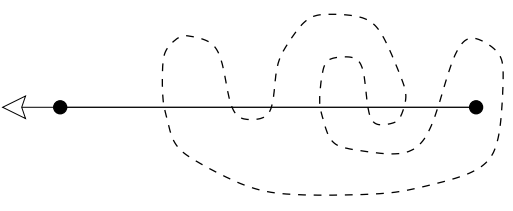

(d)

Fig. 1: Typical planar (i.e. drawn on a sphere) diagrams contributing to (a) $f_{8}(0,0)$ and $(\mathrm{c}) f_{7}(0,0)$, and one of their respective (semi-)meander pictures (b) and (d).

where $n_{1} n_{2} f_{n}\left(n_{1}, n_{2}\right)$ counts the total number of planar (genus 0 ) connected self-avoiding but mutually intersecting black and white loop configurations with $n$ intersections, weighted by their inverse symmetry factor and by a factor $n_{1}$ per black loop and $n_{2}$ per white one. Due to planarity, $f_{n}=0$ for odd $n$.

The meander numbers $M_{n}$ read

$$
M_{n}=4 n f_{2 n}(0,0),
$$

where the factor $4 n$ accounts for the $2 n$ positions between bridges on the black loop where to open it and 2 for the east-west orientation of the meander (see Fig.1 (a) and (b)). In this language, rivers correspond to black loops, while roads correspond to white ones, and the limit $n_{1}, n_{2} \rightarrow 0$ simply eliminates the configurations with more than one loop of each color.

A semi-meander is nothing but a configuration of a black open segment and a white closed loop, in which one of the extremities of the black segment is sent to infinity (this is always possible in the planar case, where configurations are drawn on a sphere, as illustrated in Fig.1 (c) and (d)). In the matrix language, it corresponds to a large $N$ correlation function of the operator

$$
\phi_{1}=\lim _{N \rightarrow \infty} \frac{1}{N} \sum_{i=1}^{n_{1}} \operatorname{Tr}\left(B_{i}\right)
$$


that creates a black endpoint, namely

$$
\left\langle\phi_{1} \phi_{1}\right\rangle=\sum_{n=1}^{\infty} n_{1} n_{2} \bar{f}_{n}\left(n_{1}, n_{2}\right) x^{n}
$$

where $n_{1} n_{2} \bar{f}_{n}\left(n_{1}, n_{2}\right)$ counts the number of configurations of rivers made of one segment and a number of loops, intersecting closed roads. Again the semi-meander numbers simply read

$$
\bar{M}_{n}=2 \bar{f}_{n}(0,0)
$$

as we have picked one endpoint of the segment to send it to the infinity on the left (see Fig.1 (d)).

\section{Meanders as a height model: fully packed loop model}

The $O\left(n_{1}, n_{2}\right)$ model above is a particular version of a fully-packed loop (FPL) model on a random surface. The random surface is dual to the graphs occurring in the Feynman expansion of the free energy (2.2). By full packing, we mean that the loops visit all the vertices of the graph. Moreover, each edge is visited by either a black or a white loop.

On the regular square lattice, these two properties are characteristic of the $F P L^{2}\left(n_{1}, n_{2}\right)$ loop model of [15]. A configuration of this model is characterized by a set of fully packed black loops visiting all the vertices and half of the edges, the other half of the edges forming fully packed white loops. Each black (resp. white) loop receives a weight $n_{1}$ (resp. $n_{2}$ ). For $n_{1}=n_{2}=2$, the loop fugacities are realized by assigning independent orientations to all the loops. An oriented black and white fully packed loop configuration may be equivalently translated into a three-dimensional height configuration on the faces of the lattice as follows. We first bicolor the vertices of the lattice, by letting vertices marked with $\bullet$ and with $\circ$ alternate around each face. Next we define a vector variable on each edge according to the rule:

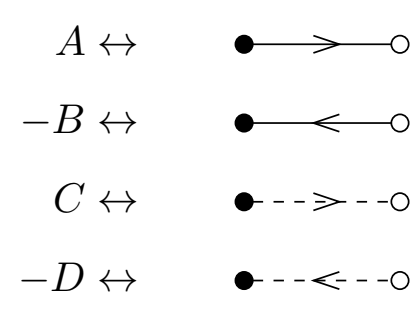


where $A, B, C, D$ are four fixed vectors with vanishing sum (hence generically in $\mathbb{R}^{3}$ ). To determine the height $h$ on each face, we use the Ampère rule, that $h$ increases (resp. decreases) by the edge vector crossed, if it points to the left (resp. right). This is well defined thanks to the relation $A+B+C+D=0$. Note in this formulation that exchanging $A \leftrightarrow-B$ (resp. $C \leftrightarrow-D$ ) along a loop amounts to reversing the orientation of the corresponding black (resp. white) loop. This defines the $F P L^{2}\left(n_{1}=2, n_{2}=2\right)$ model, which is critical. In terms of the height variable, this model is described in the continuum limit by three free fields (one for each component of the height vector), hence a conformal theory with central charge $c=3$. This model can be modified by introducing local Boltzmann weights that assign a weight $n_{1}$ resp. $n_{2}$ per loop of either kind: this is the $F P L^{2}\left(n_{1}, n_{2}\right)$ model. Remarkably, the $F P L^{2}\left(n_{1}, n_{2}\right)$ model remains critical for $n_{1}, n_{2} \leq 2$. It is still described by a 3-dimensional Coulomb gas, but with two additional electric charges at infinity, resulting in a central charge [15]:

$$
c_{F P L}\left(n_{1}, n_{2}\right)=3-6\left(\frac{e_{1}^{2}}{1-e_{1}}+\frac{e_{2}^{2}}{1-e_{2}}\right), \quad n_{i}=2 \cos \left(\pi e_{i}\right), i=1,2 .
$$

We may now define this model on a random surface, by representing its configurations as graphs made of white and black edges (for the two types of loops), and vertices of the form

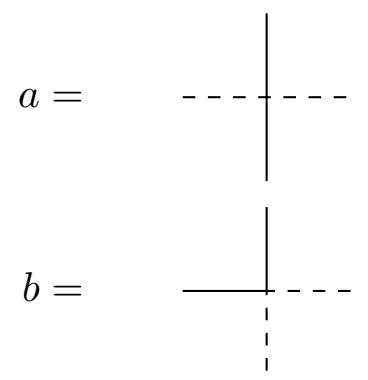

and weighting each black, resp. white, loop with a factor $n_{1}$, resp. $n_{2}$. Moreover, as we have seen above, the vertices of the graph must be bicolored with alternating marks $\bullet$ and $\circ$, and the white and black loops oriented, in order to define a unique three-dimensional edge configuration, using (3.1). On planar graphs the bicolorability of the vertices ensures that the tessellation dual to the graph is Eulerian.

If we relax this constraint of bicolorability, it is no longer possible to define threedimensional edge variables, but it is actually easy to see that the black and white loop configurations are now faithfully reproduced by considering only two edge variables, say $A$ 
for black edges and $C$ for white ones, which in turn amounts to setting $A+B=C+D=0$. With the same Ampère rule across oriented black or white edges, we see that the height $h$ becomes two-dimensional, as it takes only values of the form $h_{0}+m A+p C, m, p$ two integers. The net effect has therefore been, by lack of bicolorability of the graphs, to reduce the height variable to a two-dimensional space, resulting in

$$
\begin{aligned}
c\left(n_{1}, n_{2}\right) & =2-6 \frac{e_{1}^{2}}{1-e_{1}}-6 \frac{e_{2}^{2}}{1-e_{2}} \\
& =c\left(n_{1}\right)+c\left(n_{2}\right) \\
c(n) & =1-6 \frac{e^{2}}{1-e}, \quad n=2 \cos (\pi e) .
\end{aligned}
$$

This shift by -1 in the central charge when going from Eulerian to unconstrained tessellations has already been observed in [16]. Note also that the central charge $c\left(n_{1}, n_{2}\right)=$ $c\left(n_{1}\right)+c\left(n_{2}\right)$ is that of two decoupled free fields. In flat space, such an effective decoupling of the two a priori coupled free fields describing the FPL model has already been observed in [15].

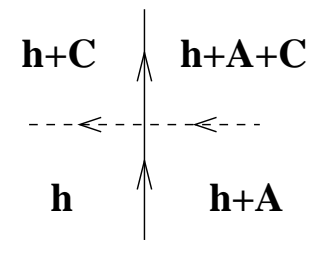

$a$
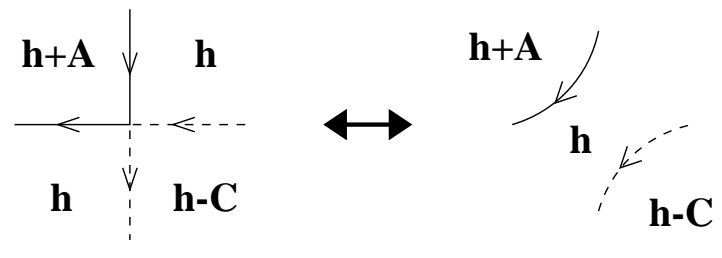

Fig. 2: Height configurations around a vertex of the $F P L^{2}\left(n_{1}, n_{2}\right)$ model on a non-bicolorable graph. Black edges correspond to the values $A=-B$, while white edges correspond to $C=-D$. In the $b$ vertex, the height on the two opposite SW and NE faces is the same, hence the vertex may be undone as shown.

The $O\left(n_{1}, n_{2}\right)$ model is a special version of this in which the type $b$ vertex of (3.3) and Fig.2 is forbidden. As shown in Fig.2, this vertex is expected to be irrelevant anyway, as the height $h$ takes the same value in the SW and NE faces, so that the vertex can be "undone" to let these two faces communicate without altering the height configuration.

It is interesting to note that for this particular case (without $b$ vertex) the bidimensionality of the height variable can be recovered in a slightly different way. Indeed, in the absence of the type $b$ vertex, the graphs are automatically bicolorable, hence allowing a 


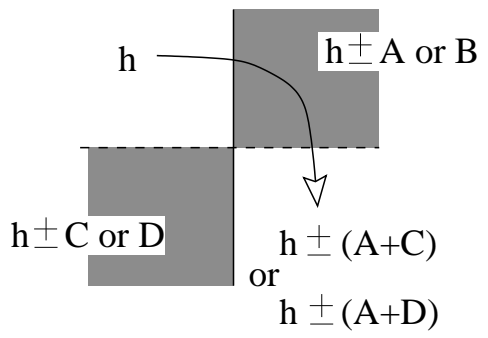

Fig. 3: Height configurations around a vertex of the $O\left(n_{1}, n_{2}\right)$ model. Black edges correspond to the values $A$ or $-B$, while white edges correspond to $C$ or $-D$. The height difference between two opposite faces may only take the values $A+C, A+D, B+C=-(A+D)$ and $B+D=-(A+C)$.

priori for the construction of a three-dimensional height. But it turns out that the heights on two opposite faces around a vertex of type $a$ may only differ by the quantities $\pm(A+C)$, $\pm(A+D)$, as illustrated in Fig.3, whereas the differences $\pm(A+B)=\mp(C+D)$ are forbidden. This means that the graph, whose faces can be naturally bicolored (say black and white), must have all the heights on white faces in the same plane generated by $A+C$, and $A+D$, and all the heights on the black faces on a parallel plane, distant by $C$ or $D$. For the sake of simplicity, we may take $B=-A$ and $D=-C$ as above, without altering the model since the differences $\pm(A+B)$ and $\pm(C+D)$ never appear here, and the heights all lie in the same plane generated by $A$ and $C$.

In conclusion, we are led to the natural conjecture that the $O\left(n_{1}, n_{2}\right)$ model is the random surface version of a critical fully packed loop model described on the square lattice by two free fields, and with central charge (3.4) above. In the particular case of meanders when $n_{1}=n_{2}=0$, i.e. $e_{1}=e_{2}=\frac{1}{2}$, we find $c=-2-2=-4$ as announced.

\section{Exact exponents from Quantum Gravity at $n_{1}=n_{2}=0$}

The above identification of the meander problem as a $c=-4$ field theory on a random sphere can be confirmed by computing various exact critical exponents of the $c=-4$ conformal theory coupled to two-dimensional gravity, as expressed through the celebrated KPZ formula [14], relating the anomalous dimensions of operators in the lattice and random surface versions of the theory.

Defining a conformal theory on a random surface, one is led to introduce a new parameter, the cosmological constant, coupled to the area of the surfaces. In our matrix language, its role is played by the parameter $x$ in (2.1), through the weight $x^{n}$, where $n$ is the total number of vertices of the random graph, as well as the total area of its dual, made 
of squares of unit area. Criticality is reached when $x \rightarrow x_{c}$, such that the free energy's behavior becomes singular, with a power law

$$
F(x) \sim\left(x_{c}-x\right)^{2-\gamma_{s t r}}
$$

where $\gamma_{s t r}$ stands for the string susceptibility exponent, related to the central charge $c$ through [14]

$$
\gamma_{s t r}(c)=\frac{c-1-\sqrt{(25-c)(1-c)}}{12}
$$

valid for all $c \leq 1$. This is immediately translated into the asymptotic behavior of the coefficients $F_{n}$ in the expansion $F(x)=\sum f_{n} x^{n}$ as

$$
f_{n} \sim \frac{\left(x_{c}\right)^{-n}}{n^{3-\gamma_{s t r}}} .
$$

In the case of meanders, we have $c=-4$, and

$$
M_{n} \sim \frac{R^{2 n}}{n^{\alpha}}
$$

where $R_{c}=1 / x_{c}$, and

$$
\alpha=2-\gamma_{s t r}(c=-4)=\frac{29+\sqrt{145}}{12}=3.42013288 \ldots
$$

This value is in agreement with the recent improved numerical estimate $\alpha=3.4206(4)$ [8].

As mentioned above, the semi-meander numbers involve the computation of a correlation function of operators inserting black endpoints. The operators of a conformal theory are known to be dressed when the theory is coupled to gravity, and their correlations have the following behavior when the cosmological constant $x$ approaches its critical value $x_{c}$ :

$$
\left\langle\phi_{m_{1}} \phi_{m_{2}} \ldots \phi_{m_{k}}\right\rangle \sim\left(x_{c}-x\right)^{\sum_{i=1}^{k} \Delta_{m_{i}}-\gamma_{s t r}-(k-2)},
$$

where $\Delta_{m}$ is the anomalous dimension of the dressed operator $\phi_{m}$, and $\gamma_{s t r}$ is as in (4.2). Furthermore, the dressed dimension $\Delta$ is related to the conformal dimension $h$ of the (undressed) operator of the conformal theory through the relation [14

$$
\Delta=\frac{\sqrt{1-c+24 h}-\sqrt{1-c}}{\sqrt{25-c}-\sqrt{1-c}} .
$$

Let us now return to the case of semi-meander numbers, given by (2.5) (2.6). The operator $\phi_{1}$ creating black endpoints actually pertains to the gravitational version of the $c=-2$ 
free field theory describing the black loops. At this stage, we can keep an arbitrary weight $n_{1}$ for the black loops, i.e. consider the $O\left(n_{1}\right)$ theory with $c\left(n_{1}\right)=1-6 e_{1}^{2} /\left(1-e_{1}\right)$, $n_{1}=2 \cos \left(\pi e_{1}\right)$. In the corresponding Coulomb gas language, the correlator $\left\langle\phi_{1} \phi_{1}\right\rangle$ corresponds to a correlation $\left\langle\psi_{1}\left(z_{1}\right) \psi_{-1}\left(z_{2}\right)\right\rangle$ of a conformal operator that creates an oriented dislocation line in the height picture between two magnetic monopoles at $z_{1}$ and $z_{2}$. The corresponding magnetic charges $\pm m$ must be corrected by an electric charge $e=e_{1}$ to restore the correct weight $n_{1}$ for the segment joining two such insertion points when this segment winds around a cylinder. In general, electro-magnetic operators with electric charge $e$ and magnetic charge $m$ have conformal dimension

$$
h_{e, m}=\frac{e\left(e-2 e_{1}\right)}{4 g}+\frac{g}{4} m^{2}
$$

provided $\left(e-e_{1}\right) m=0$, where $g$ is the coupling of the free field, with $n_{1}=-2 \cos (\pi g)$, i.e. $g=1-e_{1}$. In our case, the operator of insertion of 1 line originating from one endpoint corresponds to having $m=1 / 2$ and $e=e_{1}$ (see for instance [17]). Taking now $n_{1}=0$, i.e. $e_{1}=1 / 2$, we identify for $\psi_{ \pm 1}$ the conformal dimension

$$
h_{1}=h_{e_{1}, 1 / 2}=-\frac{e_{1}^{2}}{4\left(1-e_{1}\right)}+\frac{1-e_{1}}{16}=-\frac{3}{32}
$$

and its gravitationally dressed counterpart through (4.7):

$$
\Delta_{1}=\frac{\frac{1}{2} \sqrt{11}-\sqrt{5}}{\sqrt{29}-\sqrt{5}} .
$$

The semi-meander generating function (2.5) then reads 1

$$
\left\langle\phi_{1} \phi_{1}\right\rangle \sim\left(x_{c}-x\right)^{2 \Delta_{1}-\gamma_{s t r}}
$$

as a particular case of (4.6) and gives the semi-meander asymptotics

$$
\bar{M}_{n} \sim \frac{R^{n}}{n^{\bar{\alpha}}},
$$

where

$$
\bar{\alpha}=1-\gamma_{s t r}(-4)+2 \Delta_{1}=1+\frac{1}{24} \sqrt{11}(\sqrt{29}+\sqrt{5})=2.05319873 \ldots
$$

This value again is in agreement with the recent numerical estimate $\bar{\alpha}=2.056(10)$ of $[7]$.

1 When $n_{1}, n_{2} \rightarrow 0$, we have to pick the term proportional to $n_{1} n_{2}$ in $\left\langle\phi_{1} \phi_{1}\right\rangle$, namely compute $\lim _{n_{1}, n_{2} \rightarrow 0}\left\langle\phi_{1} \phi_{1}\right\rangle /\left(n_{1} n_{2}\right)$. By a slight abuse of notation, we still write the result as $\left\langle\phi_{1} \phi_{1}\right\rangle$. 


\section{More river geometries}

The above picture leads to many interesting results for $n_{1}=n_{2}=0$ using operators of insertion of more lines. In the matrix model formalism, we introduce

$$
\phi_{k}=\lim _{N \rightarrow \infty} \frac{1}{N} \sum_{i=1}^{n_{1}} \operatorname{Tr}\left(B_{i}^{k}\right)
$$

creating $k$ black lines from a point. The magnetic analogue of this operator has dimension

$$
h_{k}=h_{e_{1}=1 / 2, k / 2}=\frac{k^{2}-4}{32}
$$

and we get the dressed dimension

$$
\Delta_{k}=\frac{\frac{1}{2} \sqrt{8+3 k^{2}}-\sqrt{5}}{\sqrt{29}-\sqrt{5}} .
$$

We may use the operators $\phi_{k}$ to compute a class of generalized meandric numbers, corresponding to situations when the river is made of several connected segments and/or loops, for instance in the cases of the river configurations depicted in Fig.4:

$$
\begin{aligned}
& \text { "k-star" : }\left\langle\left(\phi_{1}\right)^{k} \phi_{k}\right\rangle \sim\left(x_{c}-x\right)^{\alpha_{k-s t a r}-1} \\
& \Rightarrow \quad \alpha_{k-s t a r}=k \Delta_{1}+\Delta_{k}-\gamma_{s t r}+2-k \\
& \text { "p, } p-\text { star" : }\left\langle\left(\phi_{1}\right)^{p+k-2} \phi_{p} \phi_{k}\right\rangle \sim\left(x_{c}-x\right)^{\alpha_{p, k-s t a r}-1} \\
& \Rightarrow \quad \alpha_{p, k-s t a r}=(p+k-2) \Delta_{1}+\Delta_{p}+\Delta_{k}-\gamma_{s t r}+3-p-k \\
& \text { "pentagon" : }\left\langle\left(\phi_{3}\right)^{3}\left(\phi_{1}\right)^{5}\right\rangle \sim\left(x_{c}-x\right)^{\alpha_{\text {pentagon }}-1} \\
& \Rightarrow \quad \alpha_{\text {pentagon }}=5 \Delta_{1}+3 \Delta_{3}-\gamma_{s t r}-5 \\
& \text { "cherry" : }\left\langle\phi_{1} \phi_{3}\right\rangle \sim\left(x_{c}-x\right)^{\alpha_{\text {cherry }}-1} \\
& \Rightarrow \quad \alpha_{\text {cherry }}=\Delta_{1}+\Delta_{3}-\gamma_{s t r}+1 \\
& \text { "eight" : }\left\langle\phi_{4}\right\rangle \sim\left(x_{c}-x\right)^{\alpha_{e i g h t}-1} \\
& \Rightarrow \quad \alpha_{\text {eight }}=\Delta_{4}-\gamma_{s t r}+2
\end{aligned}
$$

where again $\langle\ldots\rangle$ stands for $\lim _{n_{1}, n_{2} \rightarrow 0}\langle\ldots\rangle /\left(n_{1} n_{2}\right)$. In all these cases, the corresponding meandric numbers count the configurations of a single road crossing the connected river graph, one vertex of which we have sent to infinity. As illustrated in Fig.4, we have chosen to send the central $k$-valent vertex of the $k$-star to infinity say on the left, leaving us with a river formed of $k$ parallel half-lines (connected at infinity); in that case, the counting 

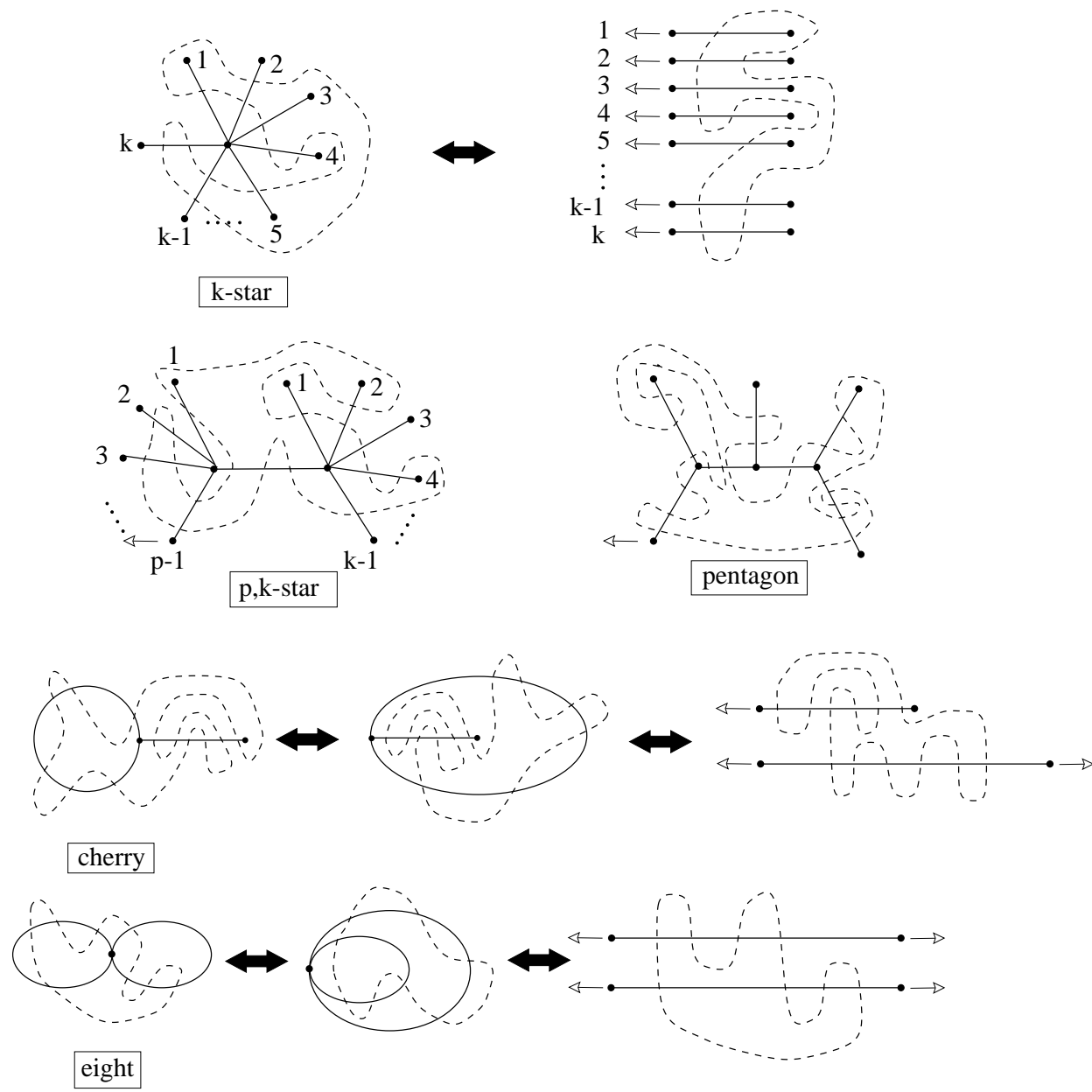

Fig. 4: Meandric numbers with particular river configurations: "k-star"=the river is a star with $k$ branches, with $k$ univalent vertices and one $k$-valent one; "p,k-star"=the river is made of two stars with respectively $p$ and $k$ branches one of which is common to both, i.e. with one $p$-valent, one $k$-valent and $p+k-2$ univalent vertices; "pentagon" =the river is a tree with three trivalent vertices and five univalent ones; "cherry"=the river is made of a loop connected to a segment, with one tri-valent and one univalent vertices; "eight"=the river is made of two loops connected at one point. On the sphere, we may send the central vertex of the river of the " $k$-star" graphs to infinity, yielding $k$ parallel semi-infinite rivers connected at infinity. Moreover, the "cherry" configuration is equivalent to that of one loop including one segment, and the connection point may be sent to infinity, yielding a semi-infinite river parallel to an infinite one. Similarly, the "eight" configuration can be transformed into that of two included loops, and the connection point may be again sent to infinity, yielding two parallel rivers (with all their ends connected at infinity).

function for theses configurations is $k\left\langle\phi_{k} \phi_{1}^{k}\right\rangle$, to account for the $k$-fold degeneracy. Note also that the "cherry" river configuration equivalently corresponds to a segment included in 
a loop, and that the connecting point can be sent to infinity, leaving us with a configuration of a semi-infinite river parallel to an infinite one, as shown in Fig.4. Similarly, the "eight" configuration corresponds to two parallel infinite rivers.

In particular, eqn.(5.4) yields for the "3-star", "pentagon", "cherry" and "eight" configurations of river, corresponding respectively to $k=3$ in the first case of (5.4), and the three last ones:

$$
\begin{aligned}
\alpha_{3-\text { star }}= & \frac{1}{2} \frac{3 \sqrt{11}+\sqrt{35}-8 \sqrt{5}}{\sqrt{29}-\sqrt{5}}+\frac{5+\sqrt{145}}{12}-1 \\
= & -1+\frac{1}{48}(\sqrt{5}+\sqrt{29})(3 \sqrt{11}+\sqrt{35}-4 \sqrt{5})=0.09899483 \ldots \\
\alpha_{\text {pentagon }}= & \frac{1}{2} \frac{5 \sqrt{11}+3 \sqrt{35}-16 \sqrt{5}}{\sqrt{29}-\sqrt{5}}+\frac{5+\sqrt{145}}{12}-5 \\
= & -5+\frac{1}{48}(\sqrt{5}+\sqrt{29})(5 \sqrt{11}+3 \sqrt{35}-12 \sqrt{5})=-3.80941298 \ldots \\
\alpha_{\text {cherry }}= & \frac{1}{2} \frac{\sqrt{11}+\sqrt{35}-4 \sqrt{5}}{\sqrt{29}-\sqrt{5}}+\frac{5+\sqrt{145}}{12}+1 \\
= & 1+\frac{1}{48}(\sqrt{5}+\sqrt{29})(\sqrt{11}+\sqrt{35})=2.46592898 \ldots \\
\alpha_{\text {eight }}= & \frac{\sqrt{14}-\sqrt{5}}{\sqrt{29}-\sqrt{5}+\frac{5+\sqrt{145}}{12}+2} \\
= & 2+\frac{1}{24}(\sqrt{5}+\sqrt{29})(\sqrt{14}+\sqrt{5})=3.89823486 \ldots
\end{aligned}
$$

In addition to the cases of Fig.4, we may also allow for disconnected rivers. For instance, we may realize a river made of $k$ distinct segments by considering, for $n_{1}, n_{2} \rightarrow 0$ :

$$
\begin{aligned}
\lim _{n_{1}, n_{2} \rightarrow 0} & \frac{1}{n_{1}^{k} n_{2}}\left\langle\left(\phi_{1}\right)^{2 k}\right\rangle \sim\left(x_{c}-x\right)^{\alpha_{k-\text { segments }}-1} \\
& \Rightarrow \alpha_{k-\text { segments }}=2 k \Delta_{1}-\gamma_{\text {str }}+3-2 k
\end{aligned}
$$

whereas we may get a river made of $k$ distinct segments, plus a $p$-star by considering

$$
\begin{aligned}
\lim _{n_{1}, n_{2} \rightarrow 0} & \frac{1}{n_{1}^{k+1} n_{2}}\left\langle\left(\phi_{1}\right)^{2 k+p} \phi_{p}\right\rangle \sim\left(x_{c}-x\right)^{\alpha_{k-\text { segments }+p-\text { star }}-1} \\
& \Rightarrow \alpha_{k-\text { segments }+p-\text { star }}=(2 k+p) \Delta_{1}+\Delta_{p}-\gamma_{s t r}+3-2 k-p
\end{aligned}
$$

In both cases (5.6) (5.7), and contrary to the situations of Fig.4 where the river is connected (possibly through the point at infinity), the road must visit all the connected components of river, to ensure that the meandric black and white graph is globally connected. 
Note finally that for $k=2$ in (5.3) we get $\Delta_{2}=0$ (this is no surprise, as $h_{2}=0$ and $\phi_{2}$ is the dressed identity operator, also called puncture operator in gravity). This means that the insertion of any number of $\phi_{2}$ in a correlation has the same effect as the same number of applications of $x \frac{d}{d x}$ on the critical asymptotics. More precisely, we have

$$
\frac{\left\langle X\left(\phi_{2}\right)^{p}\right\rangle}{\langle X\rangle} \sim\left(x_{c}-x\right)^{-p}
$$

for any combination of operators $X$. The puncture operator $\phi_{2}$ can therefore be viewed as that of marking a point on the river. In particular, we have

$$
\left\langle\phi_{2}\right\rangle \propto \sum_{n \geq 1} M_{n} x^{2 n} \sim\left(x_{c}-x\right)^{1-\gamma_{s t r}}
$$

in agreement with $\alpha=2-\gamma_{s t r}$.

\section{Discussion}

The value (3.4) of the central charge $c\left(n_{1}, n_{2}\right)$ allows us to extend the meander results to arbitrary values of $n_{1}, n_{2}$. Defining the multi-river and multi-road meander polynomial

$$
m_{n}\left(n_{1}, n_{2}\right)=4 n f_{2 n}\left(n_{1}, n_{2}\right)
$$

we have the following prediction for its large $n$ asymptotics:

$$
\begin{aligned}
m_{n}\left(n_{1}, n_{2}\right) & \sim \frac{R\left(n_{1}, n_{2}\right)^{2 n}}{n^{\alpha\left(n_{1}, n_{2}\right)}} \\
\alpha\left(n_{1}, n_{2}\right) & =2-\frac{1}{12}\left(c\left(n_{1}, n_{2}\right)-1-\sqrt{\left(25-c\left(n_{1}, n_{2}\right)\right)\left(1-c\left(n_{1}, n_{2}\right)\right)}\right) .
\end{aligned}
$$

This can be checked against the exact result [18] in the case $n_{2}=1$ and $n_{1}$ arbitrary, where $e_{2}=1 / 3$, obtained from the solution of the $O\left(n_{1}, n_{2}=1\right)$ matrix model in the limit of large size $N$. The result of 18 reads

$$
R\left(n_{1}, 1\right)=2 \frac{\sin ^{2}\left(\pi \frac{e_{1}}{2}\right)}{e_{1}^{2}}, \quad \alpha\left(n_{1}, 1\right)=2+\frac{e_{1}}{1-e_{1}}
$$

and this value agrees with our general prediction (6.2).

Note that we have no definite answer for $R\left(n_{1}, n_{2}\right)$, as the critical value $x_{c}$ of the cosmological constant is a non-universal quantity, expected to depend on $n_{1}$ and $n_{2}$ explicitly, and not just on $c\left(n_{1}, n_{2}\right)$. We expect (6.2) to hold only if

$$
c\left(n_{1}\right) \leq 1, \quad c\left(n_{2}\right) \leq 1, \quad \text { and } \quad c\left(n_{1}, n_{2}\right) \leq 1
$$


Indeed, the $O(n)$ model is no longer critical for $n>2(c(n) \leq 1$ for $n \leq 2)$, therefore we expect a different phase whenever $n_{1}>2$ or $n_{2}>2$. This phase has been investigated in the case of the gravitational $O(n)$ model [19] and found to have $\gamma_{s t r}=+1 / 2$ uniformly. Moreover, the relation (4.2) breaks down when $c>1$, and the gravity is then known [20] to degenerate in such a way that surfaces with long fingers dominate (branched polymer phase), and throughout this phase one has $\gamma_{s t r}=+1 / 2$. The corresponding value of the meander exponent is therefore $\alpha=2-\gamma_{s t r}=3 / 2$. This is in agreement with the results for the exact large $n_{2}$ expansion of the meander polynomial $n_{2} m_{n}\left(n_{1}=0, n_{2}\right)$ denoted by $m_{n}\left(n_{2}\right)$ in 6 .

The operator of insertion of one black line is still well defined and has conformal dimension $h_{1}$ given in (4.9), and dressed counterpart

$$
\Delta_{1}\left(n_{1}, n_{2}\right)=\frac{\sqrt{\frac{3}{2}\left(1-e_{1}\right)-c\left(n_{2}\right)}-\sqrt{1-c\left(n_{1}, n_{2}\right)}}{\sqrt{25-c\left(n_{1}, n_{2}\right)}-\sqrt{1-c\left(n_{1}, n_{2}\right)}} .
$$

This leads to the asymptotics of the multi-river and multi-road semi-meander polynomial $\bar{m}_{n}\left(n_{1}, n_{2}\right)=\bar{f}_{n}\left(n_{1}, n_{2}\right)$ in which the river is made of one segment and a number of loops. Recalling that the numbers $\bar{m}_{n}\left(n_{1}, n_{2}\right)$ are generated by the correlation function $\left\langle\phi_{1} \phi_{1}\right\rangle$ of the $O\left(n_{1}, n_{2}\right)$ model, we find

$$
\begin{aligned}
\bar{m}_{n}\left(n_{1}, n_{2}\right) & \sim \frac{R\left(n_{1}, n_{2}\right)^{n}}{n^{\bar{\alpha}\left(n_{1}, n_{2}\right)}} \\
\bar{\alpha}\left(n_{1}, n_{2}\right) & =\alpha\left(n_{1}, n_{2}\right)-1+2 \Delta_{1}\left(n_{1}, n_{2}\right) \\
& =1+\frac{1}{24}\left(\sqrt{25-c\left(n_{1}, n_{2}\right)}+\sqrt{1-c\left(n_{1}, n_{2}\right)}\right) \sqrt{6\left(1-e_{1}\right)-4 c\left(n_{2}\right)} .
\end{aligned}
$$

In the particular case $n_{2}=1$ (with $\left.c\left(n_{2}\right)=0\right)$, we have

$$
\bar{\alpha}\left(n_{1}, 1\right)=1+\frac{1}{24}\left(\left(2-e_{1}\right) \sqrt{\frac{6}{1-e_{1}}}+e_{1} \sqrt{\frac{6}{1-e_{1}}}\right) \sqrt{6\left(1-e_{1}\right)}=\frac{3}{2}
$$

independent of the value of $n_{1}$. This agrees with the known result for $n_{1}=0$, where $\bar{m}_{n}(0,1)=c_{n}=(2 n) ! /((n+1) ! n !) \sim 4^{n} / n^{3 / 2}$, in terms of the Catalan numbers $c_{n}$.

We expect the formula (6.6) to hold only if

$$
c\left(n_{1}, n_{2}\right) \leq 1, \quad c\left(n_{1}\right) \leq 1, \quad \text { and } \quad c\left(n_{2}\right) \leq \frac{3}{2}\left(1-e_{1}\right)
$$

The last bound corresponds to $\bar{\alpha}\left(n_{1}, n_{2}\right)=1$ in (6.6), and actually corresponds to a "winding transition" beyond which semi-meander configurations with large numbers of circles (with only one intersection with the river) dominate. 
Acknowledgements: We thank F. David for useful discussions.

Note added in proof: Since we implicitly use magnetic operators in our derivation of the exponents $\alpha$ for the various geometries, the edges of the corresponding graphs are implicitly oriented. These orientations must be such that all the edges meeting at a given vertex have the same inwards or outwards orientation. All the graphs we considered here can be equipped with such orientations except for the geometry of the "eight" and that of the "cherry". For these two cases, the apparent problem can be bypassed by marking a point on each closed loop, thus adding a bivalent vertex across which the orientation is reversed. Strictly speaking, the values of $\alpha_{\text {eight }}$ and $\alpha_{\text {cherry }}$ of Eq. (5.5) thus correspond to $\alpha_{\text {eight }}=\alpha_{\text {eight }}^{\text {marked }}+2$ and $\alpha_{\text {cherry }}=\alpha_{\text {cherry }}^{\text {marked }}+1$ where the superscript indicates that configurations are counted with a marked point on each loop. It is easy to prove that, without these marked points, the exponent for the "eight" (resp. "cherry") geometry simply reduces to the meander (resp. semi-meander) exponent, hence $\alpha_{\text {eight }}^{\text {unmarked }}=\alpha$ given by Eq. (1.2) and $\alpha_{\text {cherry }}^{\text {unmarked }}=\bar{\alpha}$ given by Eq. (1.3). 


\section{References}

[1] K. Hoffman, K. Mehlhorn, P. Rosenstiehl and R. Tarjan, Sorting Jordan sequences in linear time using level-linked search trees, Information and Control 68 (1986) 170-184.

[2] V. Arnold, The branched covering of $\mathrm{CP}_{2} \rightarrow S_{4}$, hyperbolicity and projective topology, Siberian Math. Jour. 29 (1988) 717-726.

[3] K.H. Ko, L. Smolinsky, A combinatorial matrix in 3-manifold theory, Pacific. J. Math. 149 (1991) 319-336.

[4] J. Touchard, Contributions à l'étude du problème des timbres poste, Canad. J. Math. 2 (1950) 385-398.

[5] W. Lunnon, A map-folding problem, Math. of Computation 22 (1968) 193-199.

[6] P. Di Francesco, O. Golinelli and E. Guitter, Meanders: a direct enumeration approach, Nuc. Phys. B 482 [FS] (1996) 497-535.

[7] O. Golinelli, A Monte-Carlo study of meanders, preprint cond-mat/9906329, to appear in EPJ B (2000).

[8] I. Jensen, Enumerations of Plane Meanders, preprint cond-mat/9910313.

[9] S. Lando and A. Zvonkin, Plane and Projective Meanders, Theor. Comp. Science 117 (1993) 227-241, and Meanders, Selecta Math. Sov. 11 (1992) 117-144.

[10] P. Di Francesco, O. Golinelli and E. Guitter, Meander, folding and arch statistics, Mathl. Comput. Modelling 26 (1997) 97-147.

[11] Y. Makeenko Strings, Matrix Models, and Meanders, Nucl.Phys.Proc.Suppl. 49 (1996) 226-237.

[12] G. Semenoff and R. Szabo Fermionic Matrix Models Int.J.Mod.Phys. A12 (1997) 2135-2292.

[13] P. Di Francesco, O. Golinelli and E. Guitter, Meanders and the Temperley-Lieb algebra, Commun.Math.Phys. 186 (1997) 1-59.

[14] V.G. Knizhnik, A.M. Polyakov and A.B. Zamolodchikov, Mod. Phys. Lett. A3 (1988) 819; F. David, Mod. Phys. Lett. A3 (1988) 1651; J. Distler and H. Kawai, Nucl. Phys. B321 (1989) 509.

[15] J. Jacobsen and J. Kondev, Field theory of compact polymers on the square lattice, Nucl. Phys. B 532 [FS], (1998) 635-688, Transition from the compact to the dense phase of two-dimensional polymers, J. Stat. Phys. 96, (1999) 21-48.

[16] E. Guitter, C. Kristjansen and J. Nielsen, Hamiltonian Cycles on Random Eulerian Triangulations, Nucl. Phys. B546 [FS] (1999) 731-750; P. Di Francesco, E. Guitter and C. Kristjansen, Fully Packed $O(n=1)$ Model on Random Eulerian Triangulations, Nucl. Phys. B549 [FS] (1999) 657-667.

[17] F. David and B. Duplantier, Exact partition functions and correlation functions of multiple Hamiltonian walks on the Manhattan lattice, J. Stat. Phys. 51, (1988) 327434 . 
[18] L. Chekhov and C. Kristjansen, Hermitian Matrix Model with Plaquette Interaction, Nucl.Phys. B479 (1996) 683-696.

[19] B. Eynard and C. Kristjansen, More on the exact solution of the $O(n)$ model on a random lattice and an investigation of the case $|n|>2$, Nucl. Phys. B466 [FS] (1996) 463-487.

[20] B. Durhuus, J. Frölich and T. Jónsson, Nucl. Phys. B240 (1984) 453-480; F. David, Nucl. Phys. B487[FS] (1997) 633-649. 